\title{
Helicos Sequencing
}

National Cancer Institute

\section{Source}

National Cancer Institute. Helicos Sequencing. NCI Thesaurus. Code C146816.

A proprietary next-generation DNA sequencing system from Helicos BioSciences based on zero wave guide technology and using unamplified DNA templates that are anchored to the surface of a glass slide. For each nucleotide cycle, polymerases are bound to the immobilized templates, and both fluorescently labeled nucleotides and proprietary terminators, which kinetically inhibit the polymerase from incorporating more than a single base, are added. The slide surface is then imaged with a high-intensity laser and where the fluorescent signal detected indicates a base incorporation at that position in the fluorescing template sequence. 